\title{
Identificação de Situações de Risco para Pacientes em Reabilitação Cardíaca Explorando uma Arquitetura de Software na Internet das Coisas
}

\author{
Douglas Scheunemann ${ }^{1}$, Adenauer Yamin ${ }^{1}$, João Lopes ${ }^{2}$, Cláudio Geyer ${ }^{2}$ \\ ${ }^{1}$ Universidade Católica de Pelotas (UCPEL) \\ Pelotas - RS - Brasil \\ ${ }^{2}$ Universidade Federal do Rio Grande do Sul (UFRGS) \\ Porto Alegre - RS - Brasil \\ scheunemann.d.a@gmail.com, adenauer.yamin@ucpel.edu.br \\ $\{j l b l o p e s$, geyer\}ainf.ufrgs.br
}

\begin{abstract}
The Internet of Things (IoT) is influencing how computer systems are developed, enabling new application scenarios and a more proactive user interaction, expanding features of mobility and availability. In this work the infrastructure provided by IoT is applied in an architecture to identify risk situations for patients in cardiac rehabilitation. The proposed architecture, called EXEHDA-HP, supports hybrid processing of context data combining techniques based on specification and learning to identify situations.
\end{abstract}

Resumo. A Internet das Coisas (IoT) vem influenciando a maneira como os sistemas computacionais são desenvolvidos, possibilitando novos cenários de aplicação e uma interação mais proativa com os usuários, expandindo características de mobilidade e disponibilidade. Neste trabalho a infraestrutura oferecida pela IoT é explorada por uma arquitetura na identificação de situações de risco para pacientes em reabilitação cardíaca. A arquitetura proposta, denominada EXEHDA-HP, suporta o processamento híbrido de contexto combinando o emprego de técnicas baseadas em especificação e aprendizado para a identificação de situações.

\section{Introdução}

Avanços em tecnologias de sensores, atuadores e protocolos de rede sem fio tem permitido o desenvolvimento de soluções de computação embarcada para diferentes finalidades. Dispositivos como utensílios domésticos, carros, equipamentos médicos e smartphones podem ser conectados e interagir a qualquer momento, em qualquer lugar com qualquer pessoa ou outro dispositivo através de uma rede unificada, denominada de Internet das Coisas (IoT) [Razzaque et al. 2015].

A principal contribuição deste trabalho é uma arquitetura de software, integrada ao middleware EXEHDA (Execution Environment for Highly Distributed Applications) [Lopes et al. 2014a], chamada EXEHDA-HP (EXEHDA - Hybrid Processing), a qual provê ciência de situação para aplicações em IoT, permitindo o processamento híbrido de dados de contexto, combinando técnicas baseadas em especificação e aprendizado para detecção de situações. Esta combinação provida pela abordagem híbrida, contribui para qualificar o mecanismo de detecção de situações [Ye et al. 2012]. 
Através do estudo de caso em reabilitação cardíaca foi possível identificar o potencial de uso da arquitetura proposta em aplicações médicas que necessitem de funções para ciência de situação no cenário distribuído típico da Internet das Coisas.

\section{EXEHDA-HP: Concepção da Arquitetura}

O middleware EXEHDA possui dois tipos de servidores: (i) Servidor de Borda, responsável por interagir com ambiente através de sensores e atuadores; e (ii) Servidor de Contexto, responsável por prover funcionalidades para ciência de situação. Estes servidores são alocados em células no ambiente gerido pelo EXEHDA, onde cada célula possui um Servidor de Contexto e pode possuir vários Servidores de Borda.

O Servidor de Contexto é formado por cinco módulos: Aquisição, Atuação, Notificação, Comunicação e Processamento [Lopes et al. 2014b]. A arquitetura do EXEHDA-HP, apresentada na Figura 1, está integrada ao Módulo de Processamento.

O bloco Gerenciador de Contexto é responsável pela funcionalidade de processamento dos dados contextuais. As etapas de fusão e interpretação utilizam modelos baseados em aprendizado, regras, ou ambos, caracterizando assim um modelo híbrido. Um dos principais desafios do modelo híbrido proposto é permitir que o treinamento e uso de algoritmos de aprendizado possa ser feito de uma forma transparente para as camadas de aplicação do EXEHDA. Para adicionar essa funcionalidade ao EXEHDA, foram criados os componentes do bloco Mecanismos de Aprendizado.

Utilizando a API de Treinamento o administrador da aplicação poderá disponibilizar os dados de treinamento, escolher a técnica de processamento e especificar as características que devem ser extraídas dos dados para executar a técnica de aprendizado. Após a execução do treinamento, os parâmetros obtidos são armazenados no repositório Modelos de Aprendizado. Esses parâmetros são acessados através da API de Execução durante a utilização da aplicação.

O bloco Extração de Características executa o pré-processamento das variáveis de contexto ou de sinais obtidos diretamente de sensores, extraindo o conjunto de características relevantes para o treinamento e a utilização dos algoritmos de aprendizado. O Classificador de Situações permite a inferência de situações considerando as informações de contexto geradas pelo processamento híbrido. São aplicados modelos de raciocínio fuzzy em conjunto com técnicas de aprendizado para classificação.

\section{EXEHDA-HP: Avaliação da Arquitetura}

Para realizar a avaliação das funcionalidades da arquitetura foi prototipada uma aplicação para monitoramento de pacientes em reabilitação cardíaca após um acidente vascular. Estudos na área de cardiologia indicam que exercícios físicos podem reduzir o tempo de recuperação destes pacientes. Porém, respostas desproporcionais na frequência cardíaca podem indicar situações de risco para o paciente. Desta forma, o reconhecimento da atividade física e sua correlação com a frequência cardíaca pode permitir uma recuperação mais segura [Negrão and Barreto 2010]. O risco inerente a situação do paciente foi classificado no domínio linguístico: baixo, moderado e alto.

No trabalho de Kwapisz et al. [Kwapisz et al. 2011] foram capturados sinais de acelerômetros de smartphones de voluntários durante a execução das seguintes atividades 


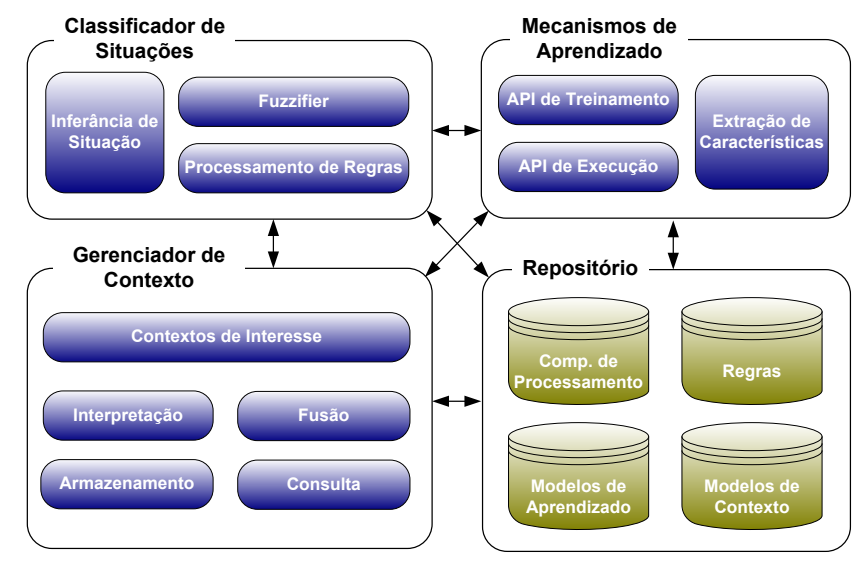

Figura 1. Arquitetura do EXEHDA-HP

físicas: caminhando, correndo, subindo escada, descendo escada, sentado e de pé. Os sinais foram disponibilizados em um banco de dados, o qual foi utilizado neste trabalho para verificar o funcionamento dos mecanismos de aprendizado do EXEHDA-HP na classificação de atividades.

Os resultados de classificação de atividades para diferentes algoritmos de aprendizado podem ser vistos na Tabela 1. Dentre os métodos, o que apresentou a maior exatidão geral foi Árvore de Decisão J48. Além disso, esse método também necessitou um menor tempo de processamento para seu treinamento.

Tabela 1. \% de atividades corretamente classificadas.

\begin{tabular}{ccccc}
\hline & Árvore de Decisão J48 & Redes Bayesianas & Redes Neurais & \multirow{2}{*}{ Regressão } \\
\hline Caminhando & $87.33 \%$ & $82.94 \%$ & $89.23 \%$ & $92.37 \%$ \\
Correndo & $95.80 \%$ & $94.19 \%$ & $98.89 \%$ & $97.41 \%$ \\
Subindo Escadas & $59.21 \%$ & $39.31 \%$ & $52.96 \%$ & $24.51 \%$ \\
Descendo Escadas & $54.66 \%$ & $30.64 \%$ & $40.17 \%$ & $10.35 \%$ \\
Sentado & $98.66 \%$ & $94.98 \%$ & $98.33 \%$ & $95.32 \%$ \\
De Pé & $97.56 \%$ & $84.96 \%$ & $93.90 \%$ & $91.06 \%$ \\
Total & $84.88 \%$ & $77.50 \%$ & $84.39 \%$ & $79.05 \%$ \\
\hline \hline
\end{tabular}

A frequência cardíaca apropriada para cada atividade física deve ser previamente informada pelo terapeuta. O domínio linguístico para a frequência cardíaca é dado por: muito baixa, baixa, normal, alta e muito alta. No bloco Fuzzifier os valores numéricos obtidos do sensor de frequência cardíaca são transformados para o domínio fuzzy aplicando uma função de pertinência trapezoidal. Na Figura 2, é mostrado o algoritmo de raciocínio fuzzy aplicado para determinar a situação que representa o risco atual do paciente.

\section{Trabalhos Relacionados}

O estudo de trabalhos relacionados foi feito considerando as especificações do EXEHDAHP: arquitetura utilizada em diferentes domínios de aplicação; arquitetura distribuída; modelo híbrido de processamento de contexto; suporte para treinamento de algoritmos de aprendizado e identificação de situações.

Yuan e Herbert [Yuan and Herbert 2014] propõem uma arquitetura para suporte ao monitoramento reconhecimento de atividades e hábitos. Neste trabalho é utilizado apenas um tipo de algoritmo de aprendizado, o qual é baseado em casos. No EXEHDA- 
HP quatro algoritmos de aprendizado foram testados, além disso, é oferecido suporte para inclusão de novos algoritmos no repositório de componentes de processamento.

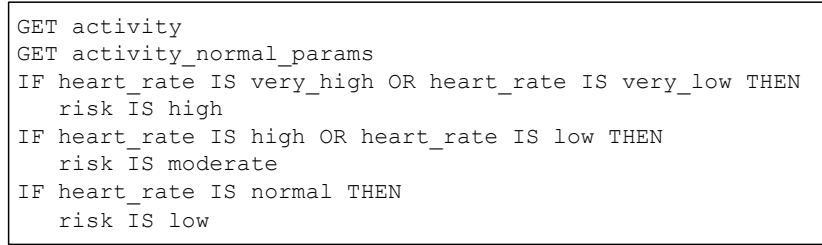

Figura 2. Algorítimo para inferência de situação

Haghighi et al. [Haghighi et al. 2014] apresentam uma arquitetura para uma aplicação específica, sem suporte a processamento distribuído. Já o EXEHDA-HP foi especificado para ser independente do domínio de aplicação e sua arquitetura foi construída para poder operar de forma distribuída.

\section{Considerações Finais}

A principal contribuição do EXEHDA-HP é uma arquitetura para prover ciência de situação, com suporte ao processamento híbrido de dados de contexto, aplicando técnicas baseadas em especificação e aprendizado para detectar situações.

Como trabalho futuro pretende-se otimizar as rotinas de treinamento dos algoritmos de aprendizado, incluindo duas novas funções: seleção automática de características e seleção automática de técnica de aprendizado.

\section{Referências Bibliográficas}

Haghighi, D. P., Averi, P., Indrawan-Santiago, M., and Minh, H. T. (2014). Situationaware mobile health monitoring. ICST (Institute for Computer Sciences, SocialInformatics and Telecommunications Engineering), pages 248--256.

Kwapisz, J. R., Weiss, G. M., and Moore, S. a. (2011). Activity recognition using cell phone accelerometers. ACM SIGKDD Explorations Newsletter, 12:74.

Lopes, J., Souza, R., Geyer, C., Costa, C., Barbosa, J., Pernas, A., and Yamin, A. (2014a). A middleware architecture for dynamic adaptation in ubiquitous computing. Journal of Universal Computer Science, 20(9):1327-1351.

Lopes, J., Souza, R., Pernas, A., Yamin, A., and Geyer, C. (2014b). A distributed architecture for supporting context-aware applications in ubicomp. In Advanced Information Networking and Applications (AINA), 2014 IEEE 28th International Conference on, pages 584-590.

Negrão, C. E. and Barreto, A. C. P. (2010). Cardiologia do Exercício: do Atleta ao Cardiopata. Manole, Barueri, SP - Brazil, 3 edition.

Razzaque, M. A., Milojevic-Jevric, M., Palade, A., and Clarke, S. (2015). Middleware for Internet of Things: a Survey. Internet of Things Journal, IEEE, PP(99):1.

Ye, J., Dobson, S., and McKeever, S. (2012). Review: Situation identification techniques in pervasive computing: A review. Pervasive Mob. Comput., 8(1):36-66.

Yuan, B. and Herbert, J. (2014). Context-aware hybrid reasoning framework for pervasive healthcare. Personal and Ubiquitous Computing, 18(4):865-881. 